\title{
Room -Temperature Paramagnetism of ZnO:Mn Films Grown by RF-sputtering
}

\author{
F. Oliveira, M. F. Cerqueira, M. I. Vasilevskiy, T. Viseu, J. Ayres de Campos, A. G. Rolo, \\ J. S. Martins*, N. A. Sobolev* ande. Alves** \\ Centro de Física, Universidade do Minho, Campus de Gualtar, 4710-057 Braga, Portugal \\ * I3N and Departamento de Física, Universidade de Aveiro, 3810-193 Aveiro, Portugal \\ ** ITN, Ion Beam Laboratory, E. N. 10, 2686-953 Sacavém, Portugal
}

Contact author: fcerqueira@ fisica.uminho.pt

Keywords: $\mathrm{ZnO}$; Mn doping; magnetic resonance, Faraday effect

\begin{abstract}
ZnO}: \mathrm{Mn}$ transparent thin films $(d<1 \mu \mathrm{m})$ with the Mn contents ranging from 1.8 to 3.25 atomic \% were grown by RF magnetron co-sputtering. The films are nanocrystalline, with wurtzite-structure grains of a typical size of $20 \mathrm{~nm}$ and with a preferential orientation of the $c$-axis perpendicular to the surface. According to the Raman spectroscopy data, Mn mostly substitutes $\mathrm{Zn}$ in the lattice sites. In spite of these factors, the nanostructure and the $\mathrm{Mn}(\mathrm{Zn})$ substitution, that are considered favorable for ferromagnetism in this material, both magnetic resonance and Faraday effect measurements show paramagnetic behavior of the $\mathrm{ZnO}: \mathrm{Mn}$ films and absence of ferromagnetic order at room temperature.
\end{abstract}

\section{Introduction}

Since the famous prediction [1] that $\mathrm{ZnO}$, along with other nonmagnetic oxide semiconductors and insulators [2] should become ferromagnetic at room temperature (RT) after doping with just a few percent of Mn (below the solubility limit), it has become a potentially very interesting material for spintronics, simultaneously magnetic, semiconducting and optically transparent. Numerous studies of $\mathrm{ZnO}: \mathrm{Mn}$ produced by a 
variety of techniques, undertaken so far, have not been able to convincingly confirm this prediction, even though there are several reports in the literature appointing to the ferromagnetism (FM) of $\mathrm{ZnO}: \mathrm{Mn}$ thin films at RT [3, 4] or below it [5]. An opinion has been expressed [6] that the FM of $\mathrm{ZnO}$ films doped with magnetic ions, when observed, is unrelated to the ions and is rather due to native defects like oxygen vacancy, similar to other diamagnetic oxides like $\mathrm{TiO}_{2}, \mathrm{HfO}_{2}$ and $\mathrm{In}_{2} \mathrm{O}_{3}$ [7]. Such defects, of course, exist already in undoped materials, although the doping can enhance their formation [6]. However, surfaces and grain boundaries are more important than the doping, in the opinion of the authors of Refs. [6,7], so the ferromagnetism can be observed only in very thin films or nanostructures. Recently, an extensive study [8] was published, based on an analysis of the data from the literature and new experiments, suggesting that grain boundaries and related vacancies are the intrinsic origin for RT ferromagnetism in $\mathrm{ZnO}$. Based on this conclusion, nanograined pure and $\mathrm{Mn}$-doped $\mathrm{ZnO}$ films were prepared, which have revealed reproducible FM at RT, even for pure $\mathrm{ZnO}$ films [8]. Inspired by these findings, we have undertaken a new attempt to achieve the FM in nanocrystalline $\mathrm{ZnO}$ thin films grown by $\mathrm{RF}$ co-sputtering with different $\mathrm{Mn}$ fraction. In this communication, we present, besides the characterization information, our experimental results on the RT magnetic properties of the films obtained by means of the magnetic resonance (MR) studies and Faraday effect (FE) spectroscopy.

\section{Experimental Details}

$\mathrm{ZnO}: \mathrm{Mn}$ thin films were grown on glass substrates in an Alcatel SCM 650 sputtering system, within a mixture of $\mathrm{O}_{2}$ and $\mathrm{Ar}$ gases (relative oxygen fraction 0.23), at a constant working pressure of $0.7 \mathrm{~Pa}$, and under either $50^{\circ} \mathrm{C}$ or $400^{\circ} \mathrm{C}$ substrate temperature. The deposition rate ranges from 1.2 to $3.4 \mathrm{~nm} / \mathrm{min}$. The target, spaced $60 \mathrm{~mm}$ away from the substrates, consisted of a hyper-pure (99.99\%) metal zinc wafer where some pieces of high purity (99.99\%) manganese were added for the Mn-doping. Radio-frequency (13.56 $\mathrm{MHz}$ ) reactive sputter deposition was carried out after the camera had reached a base pressure of $5 \times 10^{-5} \mathrm{~Pa}$. All the films were annealed under atmospheric pressure for $1 \mathrm{~h}$ at $500^{\circ} \mathrm{C}$.

All the produced samples have been characterized by means of X-ray diffraction (XRD), optical transmittance and Raman spectroscopies. XRD measurements were performed at 
RT using a Philips PW 1710 diffractometer with $\mathrm{Cu}-\mathrm{K}_{\alpha}$ radiation, in a Bragg-Bretano geometry in the range of scattering angles $10^{\circ}<2 \theta<80^{\circ}$.

The Mn content in the films was obtained by means of a combination of the Rutherford Backscattering together with Proton Induced X-Ray Emission (PIXE) experiments, using an energy of $2 \mathrm{MeV}$ and two detectors, namely, $\mathrm{Si}(\mathrm{Li})$ and Passivated Implanted Planar silicon (PIPS).

Optical transmittance spectra were measured using a Shimadzu UV 3101 PC spectrophotometer from 300 to $2500 \mathrm{~nm}$, from which the thicknesses and the band gap energy values $\left(E_{\mathrm{g}}=3.2-3.3 \mathrm{eV}\right)$ were determined. Micro-Raman spectra, taken in order to get insight into lattice vibration modes of the material, were recorded on a Jobin-Yvon T64000 spectrometer in the frequency range of 200-700 $\mathrm{cm}-1$, using the $488 \mathrm{~nm}$ excitation line of an Ar+ laser and a back-scattering geometry.

The electron magnetic resonance (FMR) measurements were performed in the $\mathrm{X}$ band $(\sim 9.5 \mathrm{GHz})$ at room temperature using a Bruker ESP 300E spectrometer. Angular dependence of the magnetic resonance signal was measured in the in-plane and out-ofplane geometry.

The Faraday effect (FE) measurements were performed using a setup including a GMW 5403FG electromagnet (magnetic field up to $1 \mathrm{~T}$ ), a Xe and/or halogen lamp, two polarizers, a Triax 320 monochromator and a UV-VIS PTM detector. The polarizers were set to form an angle of $45^{\circ}$ between their axes in order to achieve the highest resolution [9]. The Faraday rotation angle was determined by the relation, $\sin 2 \vartheta_{F}=$ $\left(I^{\uparrow}-I^{\downarrow}\right) /\left(I^{\uparrow}+I^{\downarrow}\right)$, where $I^{\uparrow}$ and $I^{\downarrow}$ are the intensities of the transmitted light for two directions of the magnetic field.

\section{Results and discussion}

\subsection{Characterization}

We studied five $\mathrm{ZnO}: \mathrm{Mn}$ films listed in Table I where their essential parameters are given. The XRD spectrum of a typical film (see Fig. 1) indicates that they are nanocrystalline having a single phase hexagonal wurtzite structure. Only (002) and (004) peaks are observed in a broad diffraction angle ranging from $10^{\circ}$ to $80^{\circ}$ due to a high preferential orientation along the $c$-axis, perpendicular to the substrate surface. The mean grain size was estimated as $D \approx 20 \mathrm{~nm}$ from the width of the (002) diffraction peak (inset in Fig. 1). With the film thickness of the order of $1 \mu \mathrm{m}$, it means that the material possesses a large 
overall area of grain boundaries and so one might expect FM in it according to the results of Ref. [8].

Table I. Samples studied in this work

\begin{tabular}{|c|c|c|c|c|c|}
\hline & $\mathrm{Mn} \# 1$ & $\mathrm{Mn \# 2}$ & $\mathrm{Mn \# 3}$ & $\mathrm{Mn \# 4}$ & $\mathrm{Mn \# 5}$ \\
\hline $\begin{array}{c}\text { Growth } \\
\text { temperature }\left({ }^{\circ} \mathrm{C}\right)\end{array}$ & 50 & 400 & 50 & 400 & 400 \\
\hline Mn (at\%) & 3.25 & 2.90 & 2.91 & 2.28 & 1.8 \\
\hline Thickness $(\mathrm{nm})$ & 722 & 854 & 907 & 1042 & 2230 \\
\hline
\end{tabular}

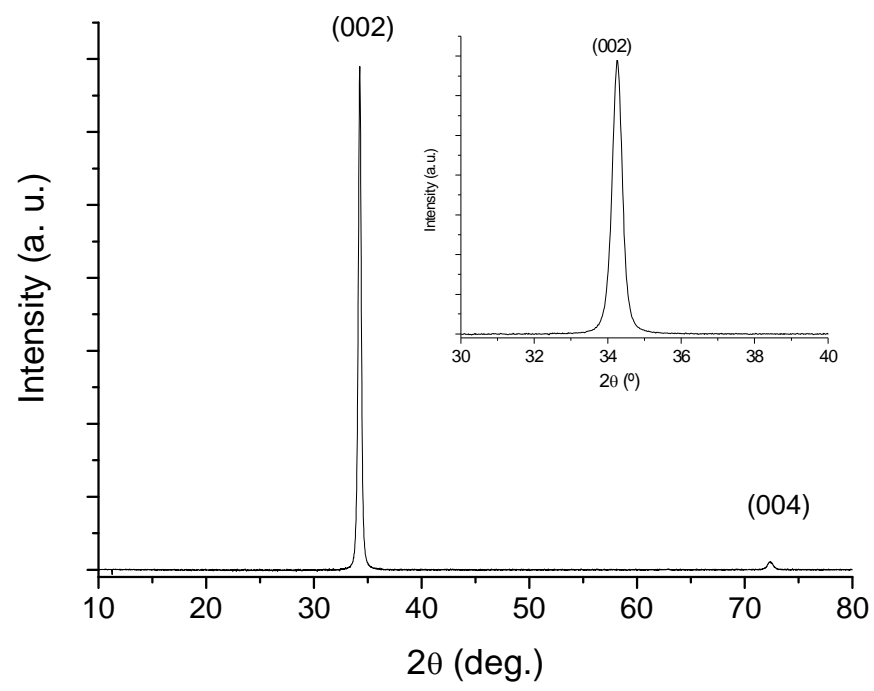

Fig. 1: Typical XRD pattern from a ZnO:Mn thin film exhibiting (002) and (004) peaks. Inset: zoom to the (002) peak.

From the Raman spectra that are presented and discussed in detail elsewhere [10], we have learn that the doping of $\mathrm{ZnO}$ with $\mathrm{Mn}$ introduces an extra band at $\approx 530 \mathrm{~cm}^{-1}$, with the intensity proportional to the Mn concentration. Such an extra band has been observed before in $\mathrm{ZnO}: \mathrm{Mn}$ doped films [11] and it has been interpreted as a local vibration mode due to $\mathrm{Mn}$ substituting $\mathrm{Zn}$ in a lattice site. Thus, we conclude that in our samples $\mathrm{Mn}$ atoms are predominantly located in the substitution positions where they should be electrically active. Then one could expect a carrier-mediated mechanism of the ferromagnetic ordering of the Mn ions as claimed in Ref. [5]. 


\subsection{Magnetic resonance}

Figure 2 shows a set of magnetic resonance (MR) curves of one of the $\mathrm{ZnO}: \mathrm{Mn}$ films, measured for different angles between the magnetic field $(B)$ and the $z$-axis (perpendicular to the film surface). From Fig. 2 it can be seen that no angular dependent signal was found. We checked that the narrow paramagnetic line at $B \approx 100 \mathrm{mT}$ has its origin in a contamination of the resonance cavity. The second narrow line at $B \approx 170 \mathrm{mT}$ appears also in the spectrum of the substrate. Only the broad band centred at approximately $350 \mathrm{mT}$ is related to the film and we attribute it to paramagnetic $\mathrm{Mn}^{2+}$ ions. The narrow lines superimposed to the broad band can be interpreted as being due to the hyperfine structure (HFS) of isolated $\mathrm{Mn}^{2+}$ ions incorporated in the $\mathrm{ZnO}$ host on $\mathrm{Zn}$ sites. In local regions with higher concentrations of magnetic ions, the linewidth increases due to the dipole-dipole interaction of the paramagnetic ions, so that a broad band appears, with the HFS of the spectra being obscured by the dipole-dipole broadening and breaks down due to the exchange interaction. Furthermore, the broad band is isotropic, with no out-of-plane angular dependence that should appear due to the demagnetization factors of a film, if the signal were of ferromagnetic origin [12].

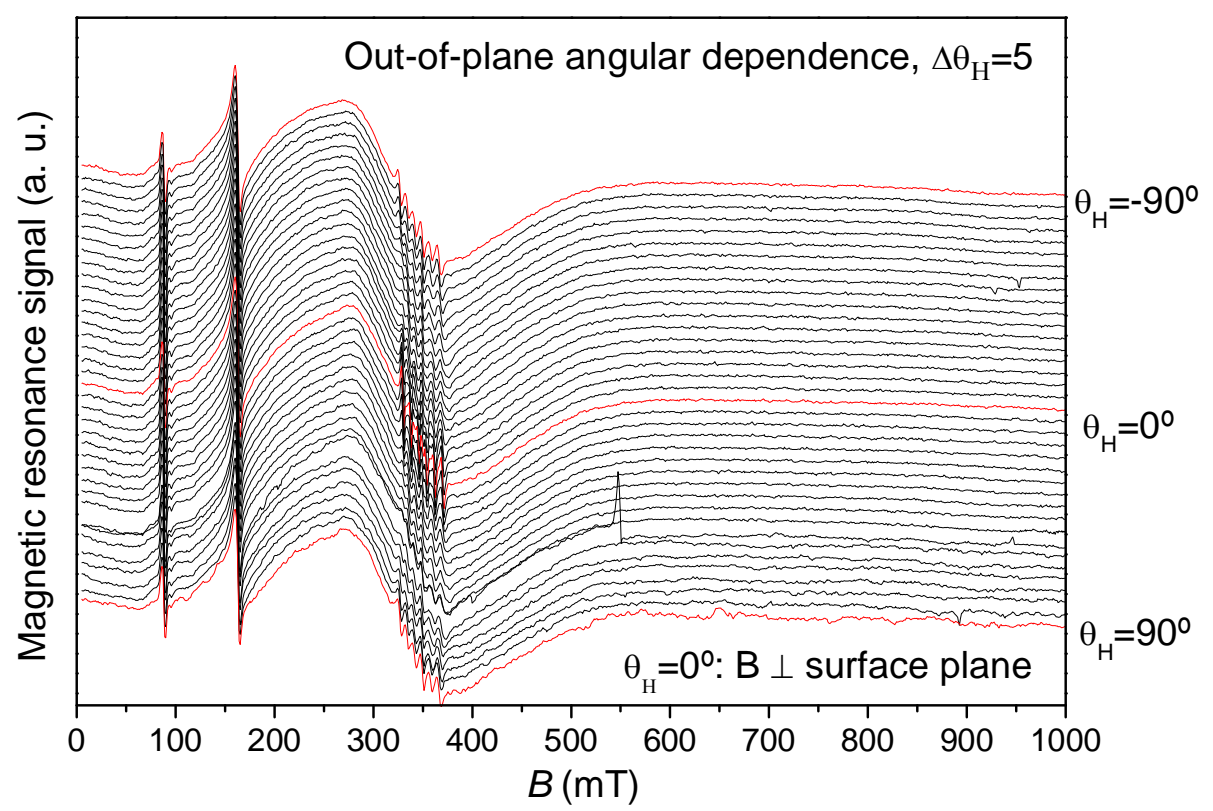


Fig. 2: (Colour online) Out-of-plane angular dependence of MR measured at room temperature for sample Mn\#5.

\subsection{Faraday effect}

The spectral dependence of the Faraday rotation angle, $\vartheta_{F}$, was measured independently for (i) the $\mathrm{ZnO}: \mathrm{Mn}$ films on glass substrates and (ii) the corresponding substrates. As a first approximation, the contribution of the film to $\vartheta_{F}$ was evaluated by subtracting the two spectra. Such a simplified procedure is justified by the fact that the interference fringes, manifest of the multiple reflections of light at the film/substrate interface, are not significant either the measured FE spectra (Fig. 3) or in the transmittance spectrum (inset to Fig. 3). The validity of this approximation was checked by the calculation of the corresponding spectra using the transfer matrix formalism [13]. The dependence of $\vartheta_{F}$ on magnetic field was found linear in the range 0-1.0 $\mathrm{T}$ in all cases. The spectra of the Verdet constant, $V=\vartheta_{F} /(B d)$, for the two films of Fig. 3 and also for Mn\#3 film are shown in Fig. 4.

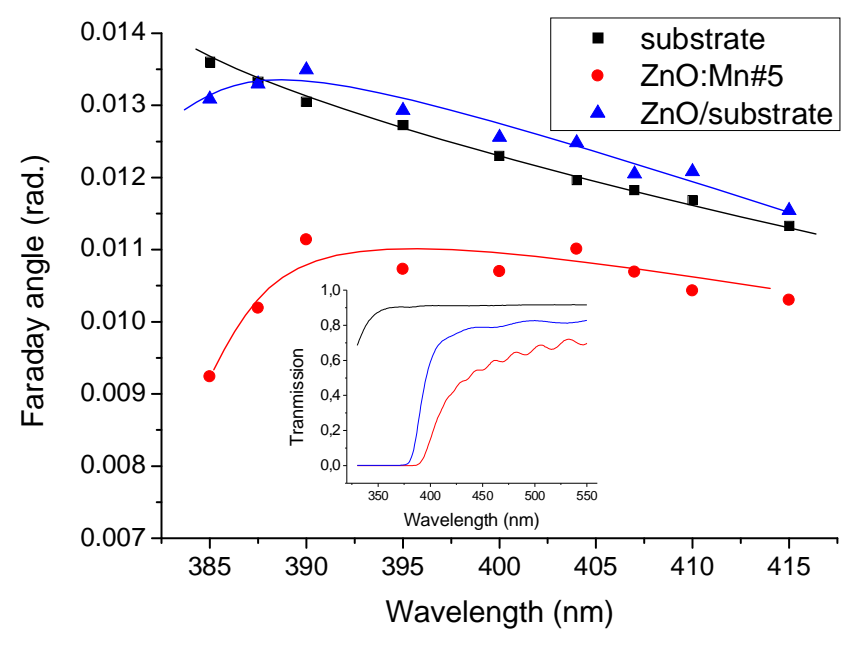

Fig. 3: (Colour online) Spectral dependence of the Faraday rotation angle for glass substrate, $\mathrm{Mn \# 5}$ sample, and undoped $\mathrm{ZnO}$ film on glass substrate. The lines are guides to the eye. Inset: transmittance spectra of the three samples. 


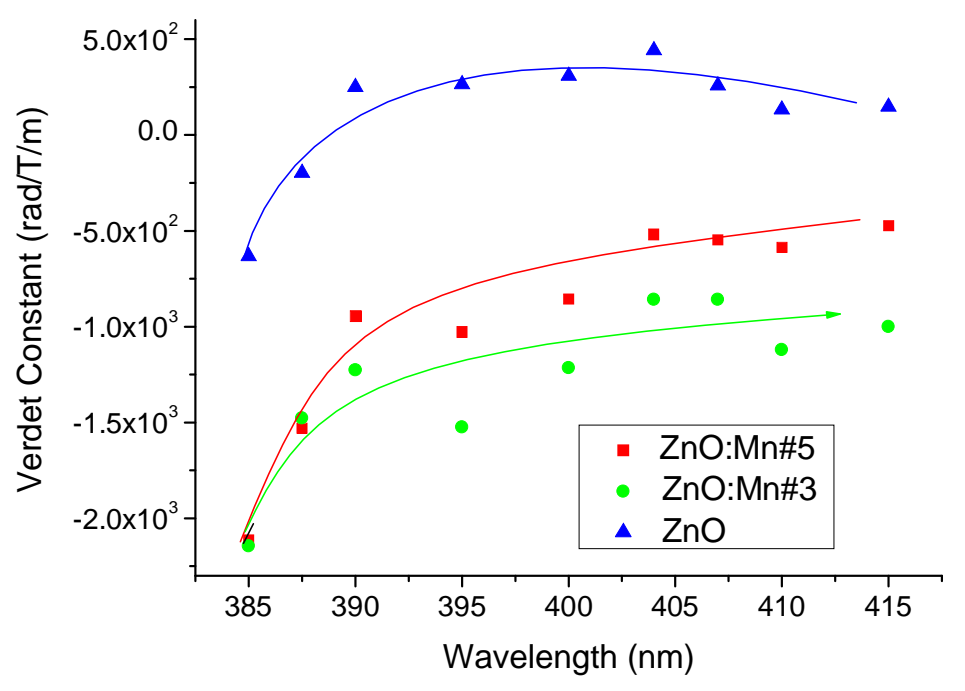

Fig. 4: (Colour online) Spectral dependence of the Verdet constant obtained from the spectra of Fig. 3 and a similar spectrum of sample Mn\#3. The lines are guides to the eye.

From Figs. 3 and 4 one can see that the FE in the ZnO:Mn film is negative $\left(\vartheta_{F}<0\right)$, which is associated with paramagnetism, while $V>0$ for pure $\mathrm{ZnO}$, in the spectral range well below the gap, which is characteristic of diamagnetic materials (indeed, pure crystalline $\mathrm{ZnO}$ is diamagnetic [14]). In the latter case, as the photon energy approaches $E_{g}$, the Verdet constant changes its sign. This is typical of non-magnetic semiconductors where the (paramagnetic) FE, in the vicinity of the gap, is associated with excitonic transitions [15]. However, the negative FE observed for the ZnO:Mn film in the whole spectral range indicates its paramagnetism due to the presence of the $\mathrm{Mn}$ ions. The complex spectral behavior of the FE spectra in Mn-doped II-VI semiconductors is attributed to several mechanisms (such as excitonic and transitions within the $3 \mathrm{~d}^{5}$ multiplet of the $\mathrm{Mn}^{2+}$ ion) acting simultaneously [15]. By comparing the spectra of samples Mn\#3 and Mn\#5 with different Mn contents (Fig. 4) we can conclude that the $\mathrm{Mn}^{2+}$ ion-related FE mechanism dominates below the gap since the shape of the spectrum is similar for both doped samples and the measured value of the Verdet constant increases with $\mathrm{Mn}$ content. When the photon energy comes quite close to $E_{g}(\approx 3.2 \mathrm{eV}$ for doped films), the samples Mn\#3 and Mn\#5 show nearly the same FE implying that a mechanism not directly related to the presence of $\mathrm{Mn}^{2+}$ ions, takes over. The maximum value of $|V|$ 
obtained by us is approximately 3 times smaller than that found for $\mathrm{Zn}_{0.9} \mathrm{Mn}_{0.1} \mathrm{O}$ single crystal in Ref. [14], which might be simply due to a smaller $E_{g}$ (higher Mn content) in the sample studied in that work. If we take into account band gap energy in the undoped sample is larger than in Mn\#3 and Mn\#5 (see inset in Fig. 3), we can guess that this mechanism also becomes stronger as $E_{g}$ is approaching in this sample (although we could not make reliable measurements beyond $385 \mathrm{~nm}$ ). Then, most likely, it involves just the inter-band optical transitions, not affected by the Mn doping.

\section{Conclusion}

In conclusion, thin transparent films of $\mathrm{Mn}$-doped $\mathrm{ZnO}$ thin were fabricated by $\mathrm{RF}$ magnetron sputtering, with $\mathrm{Mn}$ substituting $\mathrm{Zn}$ in the lattice sites. The films are nanocrystalline, with the typical grain size of $20 \mathrm{~nm}$ and high interface-to-volume ratio that should facilitate the formation of point defects. Despite these factors, favorable for the observation of RT ferromagnetism, both the magnetic resonance and Faraday rotation studies revealed the paramagnetism of our $\mathrm{ZnO}: \mathrm{Mn}$ material at $\mathrm{RT}$, similar to the results of several previous studies $[11,14]$.

\section{Acknowledgments}

The authors wish to thank the financial support from the FCT through project PTDC/FIS/72843/2006 is acknowledged.

\section{References}

1. T. Dietl, H. Ohno, F. Matsukara, J. Gilbert and D. Ferrand, Science 287, 1019 (2000).

2. S. J. Pearton, D. P. Norton, K. Ip, Y. W. Heo and T. Steiner, Prog. Mat. Sci. 50, $293(2005)$.

3. J. R. Neal, A. J. Behan, R. M. Ibrahim, H. J. Blythe, M. Ziese, A. M. Fox and G. A. Gehring, Phys. Rev. Lett. 96, 197208 (2006).

4. H. Schmidt et al, Superlattices and Microstructures 39, 334 (2006).

5. W. Shim, K. lee, W. Lee, K. A. Jeon, S. Y. Lee and M. H. Jung, J. Appl. Phys. 101, 123908 (2007) 
6. N. H. Hong, J. Sakai and V. Brizé, J. Phys.: Condens. Matter 19, 036219 (2007).

7. N. H. Hong, J. Sakai, N. Poirot and V. Brizé, Phys. Rev. B 73, 132404 (2006).

8. B. B. Straumal, A. A. Mazilkin, S. G. Protasova, A. A. Myatiev, P. B. Straumal, G. Schütz, P. A. van Aken, E. Goëring and B. Baretzky, Phys. Rev. B 79, 205206 (2009).

9. F. F. Sizov and Y. I. Ukhanov, "The Faraday and Voigt Magneto-Optic Effects Applied to Semiconductors", Naukova Dumka, Kiev, 1979 (in Russian).

10. M. F. Cerqueira et al, phys. stat. sol. (a) (submitted)

11. J. Alaria, M. Bouloudenine, G. Schmerber, S. Colis, A. Dinia, P. Turek and M. Bernard, J. Appl. Phys. 99, 08M118 (2006)

12. M. Farle, Rep. Prog. Phys. 61, 755 (1998)

13. S. Višňovský, "Optics of Magnetic Multilayers and Nanostructures", Taylor \& Francis, NY, 2006.

14. A. I. Savchuk, V. I. Fediv, S. A. Savchuk and A. Perrone, Superlattices and Microstructures 38, 421 (2005).

15. P. I. Nikitin and A. I. Savchuk, Sov. Phys. Usp. 33, 974 (1990). 